Recepción: 18 / 09 / 2016

Aceptación: 26 / 01 / 2017

Publicación: 29 / 04 / 2017

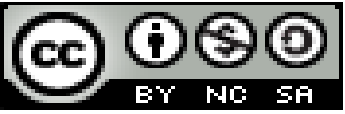

Ciencias de la salud Artículo de investigación

\title{
Factores de riesgos ocupacionales en servicios odontológicos
}

\author{
Occupational risk factors in dental services
}

\author{
factores de risco ocupacionais para serviços dentários
}

\author{
Dorys M. Bravo-Cevallos ${ }^{\mathrm{I}}$ \\ bravodorys@hotmail.com \\ Eric D. Chusino-Alarcón " \\ mg.erich0963@hotmail.es \\ Kenny M. García-Moreira ${ }^{\mathrm{II}}$ \\ kenny.garciam@gmail.com
}

\section{Correspondencia: bravodorys@ hotmail.com}

\footnotetext{
IEspecialista en Atención Primaria de la Salud, Doctor en Odontología, Docente en la Universidad Laica Eloy Alfaro de Manabí, Manta, Ecuador.

"Magister en Salud Pública Mención Gerencia de los Servicios de Salud, Diploma Superior en Educación Universitaria por Competencias, Doctor en Odontología, Docente de la Universidad Laica Eloy Alfaro de Manabí, Manta, Ecuador.

${ }^{\text {II } E s p e c i a l i s t a ~ d e ~ P r i m e r ~ G r a d o ~ e n ~ C i r u g i ́ a ~ M a x i l o ~ F a c i a l, ~ O d o n t o ́ l o g o, ~ D o c e n t e ~ d e ~ l a ~ U n i v e r s i d a d ~ L a i c a ~}$ Eloy Alfaro de Manabí, Manta, Ecuador.
} 


\section{Resumen}

La vigilancia de la salud de los trabajadores, es una actividad preventiva que sirve para proteger su salud. A tales efectos se realizó una revisión bibliográfica con el propósito de mostrar una literatura más actualizada referente a los factores de riesgo ocupacional y las enfermedades relacionadas en este ambiente.

Palabras clave: factores de riesgos; enfermedades ocupacionales; personal odontólogos.

\section{Abstract}

Surveillance of workers' health is a preventive activity that serves to protect their health. A bibliographic review was carried out with the purpose of presenting a more updated literature on occupational risk factors and related diseases in this environment.

Keywords: risk factors; occupational diseases; personal dentists.

\section{Resumo}

Monitorar a saúde dos trabalhadores, é uma atividade preventiva que serve para proteger a sua saúde. Para este efeito, uma revisão da literatura, a fim de exibir uma referência aos fatores de risco e doenças ocupacionais relacionados neste ambiente literatura mais atual foi conduzido.

Palavras chave: fatores de risco; doenças profissionais; dentistas funcionários.

\section{Introducción}

Una definición más científica afirma que "la higiene es el conjunto de conocimientos y técnicas que deben aplicar los individuos para el control de los factores que ejercen o pueden ejercer efectos nocivos sobre su salud". ${ }^{1}$

La falta de higiene puede provocar enfermedades y reacciones desfavorables no solo para los humanos, sino también para los animales y plantas, el cuidado del medio ambiente va de la mano de la higiene. ${ }^{2}$

Este artículo tiene el propósito de revisar la literatura más actualizada referente a los factores de riesgo ocupacional y las enfermedades relacionadas en este ambiente.

En este orden de ideas se puede citar la higiene del trabajo, la cual es aquella que atiende las condiciones de trabajo en cada una de las profesiones u oficios, controla los factores que puedan dañar la salud del trabajador, y adaptar el trabajo al hombre y el hombre al trabajo. ${ }^{3}$ 
Tiene por objeto, promover y mantener el más alto grado de bienestar físico, mental y social de los trabajadores de todas las profesiones, prevenir todo daño posible de ser causado en su salud, por las condiciones de trabajo protegerlos en su empleo contra riesgos resultantes de la presencia de agentes perjudiciales y colocar y mantener el trabajador en un empleo conveniente a sus aptitudes y psicológicas: fisiológicas. ${ }^{4}$

\section{Riesgos ocupacionales en odontología}

La odontología es una profesión sanitaria no exenta de riesgos para la salud de las personas que la ejercen.

Desde la declaración de la OMS, de 1946, sobre la definición de salud como «estado de absoluto bienestar físico, mental y social y no sólo como la ausencia de enfermedad», el concepto de salud ha ido variando a lo largo del tiempo. En la actualidad se considera que una persona está sana cuando tiene capacidad de producir, de ejercer el trabajo que realiza de forma cotidiana, ya sea ir a su oficina, estudiar en el colegio o universidad, realizar las labores domésticas en casa, etc. Esta capacidad de producir o trabajar es la que marca la diferencia entre una persona sana o enferma.

Además, existe una relación de la persona con el ambiente de trabajo: este ambiente va a modificar su conducta y costumbres, apareciendo una patología específica en relación directa con su trabajo. 5

Todo trabajo implica riesgos, que pueden dar lugar a:

- Enfermedades profesionales.

- Accidentes de trabajo. ${ }^{5}$

Las enfermedades profesionales u ocupacionales, son aquellas que están directamente relacionadas a la actividad laboral que desempeñamos, son el resultado a la exposición de ciertos factores de riesgo, entre las primeras están los dolores de espalda y la deformación de la columna. ${ }^{6}$

\section{Riesgos ocupacionales en odontología}


Los factores de riesgo laborales, se pueden clasificar y analizar de diferentes formas. De modo tradicional se diferencian en: físico-mecánico y no mecánico, químico, biológicos, psicosociales y ergonómico. ${ }^{7}$

En clínica odontológica se puede hablar de riesgos generales y de riesgos propios de la actividad; los primeros son asimilables a todos los patrones de riesgo, que alteran la llamada salud ocupacional. Los segundos, los propios de la actividad, se refiere a factores de riesgos determinados por ejercicio y uso de la profesión.

Riesgo físico: son producidos en el ambiente laboral por factores físicos, entre los que se pueden citar:

Traumatismos: no son tan frecuentes en esta especialidad, aunque puede citarse la proyección hacia los ojos de cuerpos extraños como el sarro o partículas minerales. ${ }^{8}$

Ruido: produce lesiones otológicas por la actuación simultánea de los factores patogenéticos fundamentales; el ruido y el tiempo de exposición. ${ }^{8}$

Con referencia a lo anterior esta profesión, no es de las más afectadas por este factor si lo comparamos con la minería, la construcción, la industria textil, entre otros.

Vibraciones: las pequeñas vibraciones recibidas normalmente en nuestra actividad, no se pueden comparar con ocupaciones en las que se emplean martillos neumáticos, sierras y taladros eléctricos, entre otros, donde las vibraciones si constituyen factores de riesgo de gran peso para la aparición de afecciones como: náuseas, vómitos, cervicalgias, sacrolumbalgias y neuropatías periféricas. $^{8}$

Iluminación: este factor repercute sobre la salud. Si es por defecto puede contribuir a la aparición de miopía, lagrimeo, fatiga visual, hiperemia conjuntival y astenopatía muscular, si por el contrario hay exceso de luz, puede aparecer el coloma central y las lesiones retinarias irreversibles. $^{8}$

La conocida luz halógena produce daño ocular, al poseer un rango de longitud de onda no atenuado por los filtros naturales del ojo. Actualmente no se le ha dado la trascendencia que merece el efecto nocivo producido y tampoco existe protección adecuada para los pacientes. ${ }^{8}$ 
Temperatura y humedad: una temperatura agradable contribuirá al mejor trabajo del equipo estomatológico. Si la temperatura es alta y el aire está saturado de humedad hay agotamiento, aumento de la temperatura corporal, puede haber trastornos respiratorios y circulatorios. ${ }^{8}$

Ventilación: debe asegurar las mejores condiciones atmosféricas posibles de temperatura, humedad, pureza y movimiento, procurando que el ambiente de trabajo sea agradable. Su déficit puede traer síntomas similares a los anteriores. ${ }^{8}$

Radiaciones ionizantes: se basa en la utilización de los equipos de Rx. Sus radiaciones actúan sobre la salud y provocan en piel: radiodermitis y la caída del cabello, sobre los ojos: cataratas.

Además, puede provocar esterilidad, anemia aplásica y leucemia, aumentando en algunos casos la incidencia de tumores malignos. ${ }^{8}$

Medio ambiente electromagnético (MAE): la creciente utilización de equipos eléctricos y electrónicos a nivel industrial residencial, comercial, en las comunicaciones y la medicina ha traído consigo una fuerte alteración del MAE natural lo que, comúnmente, se denomina contaminación electromagnética. ${ }^{8}$

Entre los equipos eléctricos o electrónicos usados frecuentemente en odontología, se encuentran las lámparas de iluminación, compresores, equipos de $\mathrm{Rx}$, sillas con mecanismos eléctricos de ajuste, unidades de cirugía, equipos de computadoras y las lámparas de fotocurado etc, no existe conciencia de los riesgos asociados a los CEM y estos equipos. Se ha investigado que campos de 50-60 hz y de solo 3 miliwatt resultan ya claramente relacionados con el cáncer. Se debe tener en cuenta el tiempo de exposición al campo, frecuencia y distancia. ${ }^{8}$

Riesgo químico: las sustancias químicas que al ponerse en contacto con el individuo puede producir las intoxicaciones ocupacionales, se producen por penetración del agente químico por tres vías: la vía respiratoria, mediante la inhalación. La vía digestiva, es menos frecuente y la piel y mucosas, en el caso de sustancias liposolubles. ${ }^{8}$

Algunos materiales dentales que pueden causar dificultades:

El yeso, formol, y otros antisépticos derivados del fenol, anestésicos locales, resinas acrílicas y compuestas, eugenol. ${ }^{8}$ 
- Primers dentales puede ocasionar dermatitis de contacto, su mayor factor de riesgo se da porque los guantes látex son permeables a estas soluciones. Los signos y síntomas más frecuentes se presentan cuando la piel entra en contacto con estas sustancias. ${ }^{8}$

- Mercurio: se deposita en órganos ricos en lípidos como el cerebro, hígado y riñón. Cuando sus niveles están por encima de los valores límites permisibles se comienzan a presentar diferentes síntomas y signos como: temblor, excitabilidad, debilidad, escalofrío, irritabilidad, angustia, náuseas, vómitos, sabor metálico, parotiditis química, tialismo, hematuria, anorexia, astenia, sensación de opresión en el tórax, daños irreversibles en el riñón, alteraciones del SNC y finalmente la muerte. Entre los exámenes realizados al personal odontológico anualmente, está la prueba biológica de concentración en orina por el método colorimétrico visual, el método espectrofotométrico de absorción atómica y la determinación de las concentraciones de mercurio en el aire de la zona de trabajo y la biopsia externa del cabello. ${ }^{8}$

- Alergia a los guantes látex: se presenta con prurito y rubor, hinchazón por contacto local directo. ${ }^{8}$

Riesgo biológico: incluye afecciones bacterianas, virales, hongos y demás enfermedades parasitarias que pueden causar enfermedades. Es importante tener en consideración tanto las enfermedades transmisibles como sus vías de transmisión. ${ }^{8}$

Riesgo ergonómico: en estomatología pueden ser abordadas desde el punto de vista ergonómico las condiciones físicas del medio, diseño de equipos adecuados que contemplen los requisitos antropométricos necesarios y evitar los vicios de posiciones, así como garantizar secuencias de movimientos lógicos y evitar la pérdida innecesaria de tiempo. ${ }^{8}$

Los riesgos antes mencionados le confieren al odontólogo un mayor o menor grado de susceptibilidad a contraer determinadas enfermedades, como son: trastornos de la estática vertebral a consecuencia paciente-operador, deformaciones de la caja toráxica, las várices y epicondilitis. ${ }^{8}$

Riesgo psicológico: el estrés que es la consecuencia de nuestra reacción interna, ante las cosas que nos suceden y las exigencias que debemos cumplir. Tiene dos componentes: los 
acontecimientos y exigencias externos impuestos en nuestras vidas y nuestra reacción interna ante ellos. $^{8}$

\section{Lesiones y enfermedades habituales}

A menudo los trabajadores no pueden escoger y se ven obligados a adaptarse a unas condiciones laborales mal diseñadas, que pueden lesionar gravemente las manos, las muñecas, las articulaciones, la espalda u otras partes del organismo. Concretamente, se pueden producir lesiones a causa de:

- El empleo repetido a lo largo del tiempo de herramientas y equipo vibratorios, por ejemplo: en la limpieza de caries y la preparación de cavidades.

- Herramientas y tareas que exigen girar la mano con movimientos de las articulaciones, por ejemplo las extracciones.

- La aplicación de fuerza en una postura forzada.

- La aplicación de presión excesiva en partes de la mano, la espalda, las muñecas o las articulaciones.

- Trabajar con los brazos extendidos o por encima de la cabeza.

- Trabajar echados hacia adelante.

- Levantar o empujar cargas pesadas.

Normalmente, las enfermedades profesionales se desarrollan lentamente.

Las enfermedades provocadas por herramientas y lugares de trabajos mal diseñados o inadecuados, se desarrollan habitualmente con lentitud a lo largo de meses o de años. Ahora bien, normalmente un odontólogo tendrá señales y síntomas durante mucho tiempo que indiquen que hay algo que no va bien. Así, por ejemplo, el odontólogo se encontrará incómodo mientras efectúa su labor asistencial o sentirá dolores en los músculos o las articulaciones una vez en casa después del trabajo. Además, puede tener pequeños tirones musculares durante bastante tiempo. 
Es importante investigar los problemas de este tipo, porque lo que puede empezar con una mera incomodidad puede acabar en algunos casos en lesiones o enfermedades que incapaciten gravemente. ${ }^{9}$

Enfermedades profesionales más frecuentes en el colectivo de odontólogo.

- Desórdenes músculo-esqueléticos.

- Contagio de enfermedades por inoculación directa o contacto.

- Hepatitis B.

- Hepatitis C.

- Síndrome de inmunodeficiencia adquirida (SIDA).

- Tétanos.

- Herpes simple.

- Contagio de enfermedades por vía inhalatoria o saliva:

-Mononucleosis infecciosa.

- IVTRS (infecciones víricas del tracto respiratorio superior)

- Infección por Helicobacter Pylori.

- Tuberculosis.

- Lesiones oculares: vista cansada, desarrollo de cataratas por la luz de polimerización.

- Patología cervical: cervialgias, dorsalgias y lumbalgias.

- Patología del brazo y hombro: epicondilitis y tendinitis del manguito de los rotadores.

- Tendinitis en la mano o síndrome del túnel carpiano.

- Trastorno psicológico: estrés, depresión y ansiedad. ${ }^{9}$

Diversos estudios, demuestran que las áreas en las que más frecuentemente aparecen las molestias dolorosas son: cuello $(79.4 \%)$, hombros $(58.3 \%)$, región dorsal $(50.5 \%)$ y lumbar 
(59.8\%). Todas las investigaciones coinciden en que las regiones más afectadas van a ser la cervical y lumbar, debido a la gran sobrecarga anatómica y fisiológica que sufre la columna vertebral al trabajar sentados con la espalda inclinada hacia delante. ${ }^{10}$

Según Grass Martínez Y et al ${ }^{11}$., en su estudio, sobre el efecto de ruido en los trabajadores del servicio estomatológico identifican los niveles de ruido existentes en el ambiente laboral de dicho servicio. En la serie se obtuvo que los departamentos de mayor nivel de ruido resultaron ser: prótesis $(73,2 \mathrm{~dB})$, ortodoncia y periodoncia $(72,7 \mathrm{~dB})$ y conservadora $(71,2 \mathrm{~dB})$, en ese orden; asimismo, en cuanto al ruido de fondo, las mayores mediciones se registraron en los departamentos de conservadora y de prótesis (68,6 y 68,1 dB, respectivamente). Por otra parte, $62,7 \%$ del total de trabajadores presentaron problemas de hipoacusia por estar expuestos permanentemente a este agente físico negativo.

A manera de colofón, en los momentos actuales, constituye un reto consolidar avances, vencer amenazas, superar debilidades e innovar estrategias, para así lograr un desarrollo integral que genere el fomento de una vida saludable.

\section{Referencias bibliográficas}

1- Higiene y salud. 2007. [citado 8 Agosto 2016]. Disponible en: http://www.educando.edu.do/articulos/generico/higiene-y-salud/

2- Definición de higiene. 2015. [citado 8 Agosto 2016]. Disponible en: http://conceptodefinicion.de/higiene/

3- Tipos de higiene 2016. [citado 8 Agosto 2016]. Disponible en: http://www.tiposde.org/salud/565-tipos-de-higiene/

4- Carrión Bolaños J. Riesgos para la salud en profesionales de la odontología. 2012. [citado 8 Agosto 2016]. Disponible en: http://www.gacetadental.com/2012/01/riesgos-para-la-salud-enprofesionales-de-la-odontologia-24896/

5- Enfermedades profesionales u ocupacionales. 2014 [citado 8 Agosto 2016]. Disponible en: http://www.info7.mx/vidayestilo/enfermedades-profesionales-u-ocupacionales/1545144 
6- Reyes Sigarreta M, Sanchez Santos L, Del Puerto Quintana C, Trujillo Mera M, Moreno Carbajal C, Cruz Acosta C. Determinante de estado de Salud capítulo 14. Página 265. Editorial Ciencias Médicas 2004. La Habana. Cuba.

7- Enfermedades profesionales del odontólogo. [citado 8 Agosto 2016]. EcuRed. Disponible en: https://www.ecured.cu/Enfermedades_profesionales_del_Odont\%C3\%B3logo

8- Espeso Nápoles Nelia, Travieso Gutiérrez Yusimí, Martínez Padilla Silvia, Puig Ravinal Luis. Factores de riesgo profesional en estomatología. AMC. 2002 [citado 8 agosto 2016]; 6(1): 9-18. Disponible en: http://scielo.sld.cu/scielo.php?script=sci_arttext\&pid=S1025$02552002000100002 \& \operatorname{lng}=\mathrm{es}$

9- Enfermedades profesionales en la odontología. Enfermedades profesionales en la odontología. 2017. [citado 23 agosto 2016]. Abogados laboralistas expertos en enfermedades profesionales en el colectivo de odontólogos y dentistas. Disponible en: https://www.abogalaboral.com/enfermedades-profesionales-en-la-odontologia.e5832

10- Mariquez Chávez J. Odontología ocupacional y enfermedades del trabajo. 2014. [citado 23 agosto 2016]. Disponible en: https://es.slideshare.net/jorgemanriquechavez/odontologaocupacional-y-enfermedades-del-trabajo

11-Grass Martínez, Y Castañeda Deroncelé M, Pérez Sánchez G, Rosell Valdenebro L, Roca Serra L. El ruido en el ambiente laboral estomatológico. Medisan 2017 [citado 23 agosto 2016]; 21(5). Disponible en: http://www.medisan.sld.cu/index.php/san/article/view/1543/html 